\title{
Modeling RF waves in spatially dispersive inhomogeneus plasma using an iterative wavelet spectral method
}

\author{
Pablo Vallejos ${ }^{1, *}$, Thomas Johnson ${ }^{1}$, and Torbjörn Hellsten ${ }^{1}$ \\ ${ }^{1}$ KTH Royal Institute of Technology, Teknikringen 29, 10044 Stockholm, Sweden
}

\begin{abstract}
The wave equation for a spatially dispersive inhomogeneous magnetized plasma is given by an integro-differential equation. The effects caused by spatial dispersion in the directions perpendicular and parallel to the magnetic field are quite different. In this study, we show how to solve the wave equation using a newly developed iterative wavelet spectral method for two cases. In the first case, the method is applied to a propagating kinetic Alfvén wave in the perpendicular direction and solved to all orders in FLR. To conserve the kinetic energy flux, first order corrections in equilibrium gradients are used in the dielectric response tensor. In the second case, we verify the method for a fast wave minority heating scenario and study the up- and downshift in the parallel wave number.
\end{abstract}

\section{Introduction}

Magnetized plasma exhibit spatial dispersive effects both in the perpendicular and parallel directions. Perpendicular spatial dispersion arises due to finite Larmor radius (FLR) effects and becomes important when the perpendicular wavelength is comparable to the Larmor radius of a plasma species. Parallel spatial dispersion is related to the Doppler shifts caused by the parallel thermal velocity and is important near cyclotron resonances. The response of a spatially dispersive medium is of non-local character. If the medium is inhomogeneous the wave equation becomes an integrodifferential equation [1-6].

Common numerical methods to solve the wave equation include the finite element method (FEM), spectral methods, or combinations of the two. By expansion of the FLR effects with respect to the inhomogeneity the integro-differential equation can be approximated with a higher order differential equation that can be solved with FEM. The up- and downshift in the parallel wave vector is more difficult to account for since the resonant interactions take place over several wavelengths. Methods based on FEM typically neglect the up- and downshift in the parallel wave vector [7-8]. Fourier spectral methods can be used for solving problems with spatial dispersion. However, these methods tend to produce dense and large matrices which are time consuming to solve.

Green et al proposed a method where the dielectric response is splitted into a dispersive and non-dispersive part [9]. By using an iterative scheme, the induced current is added by means of iteration.

More recently, a new method has been proposed based on operator splitting, an iterative scheme and wavelets $[3,4]$. Operator splitting is applied to separate dispersive and non-dispersive terms in the wave equation. The dispersive terms are added by means of iteration using Anderson Acceleration scheme [10] and the spatially dispersive response is evaluated using a Morlet wavelet representation of the electric field and dielectric response [11].

The purpose of this study is to verify the method by applying it to two cases. In the first case, the method is applied to a propagating kinetic Alfvén wave in the perpendicular direction and solved to all orders in FLR. To conserve the kinetic energy flux, first order corrections in equilibrium gradients are included $[5,6]$. In the second case, we apply the method to a fast wave minority heating scenario and study the up- and downshift in the parallel wave number.

\section{Spatially dispersive wave equation for Maxwellian plasma}

The spatially dispersive wave equation for time harmonic waves in inhomogeneous media is given by

$$
\nabla \times \nabla \times \mathbf{E}-\frac{\omega^{2}}{c^{2}} K[\mathbf{E}](\mathbf{r})=i \omega \mu_{0} \mathbf{J}_{\text {ant }}(\mathbf{r}),
$$

where $K$ is an operator defined as [5,6]

$$
\begin{aligned}
K[\mathbf{E}](\mathbf{r}) & =\int \frac{d \mathbf{k}}{(2 \pi)^{3}} \int d \mathbf{r}^{\prime} \mathbf{K}(\mathbf{r}, \mathbf{k}) \mathbf{E}\left(\mathbf{r}^{\prime}\right) e^{i \mathbf{k} \cdot\left(\mathbf{r}-\mathbf{r}^{\prime}\right)} \\
& =L[\mathbf{K}(\mathbf{r}, \mathbf{k}), \mathbf{E}(\mathbf{r})]
\end{aligned}
$$

where $\mathbf{K}$ is the dielectric kernel. The $L$ operator has been defined for simplicity in writing. Note that if the response is not spatially dispersive, then

$$
L[\mathbf{K}(\mathbf{r}), \mathbf{E}(\mathbf{r})]=\mathbf{K}(\mathbf{r}) \mathbf{E}(\mathbf{r}) .
$$

\footnotetext{
Corresponding author: pablova@kth,se
} 
To simplify our analysis, we have taken the limit for the parallel dielectric response, such that $E_{\|} \rightarrow 0$.

\subsection{Kinetic Alfvén wave}

For a plasma consisting of electrons and deuterium ions, the equation for a propagating kinetic Alfvén is given by

$$
\begin{gathered}
n_{\|}^{2} E_{x}(x)-L\left[K_{x x}\left(x, k_{x}\right), E_{x}(x)\right]=0, \\
K_{x x}\left(x, k_{x}\right)=1+\chi_{x x}\left(x, k_{x}\right),
\end{gathered}
$$

where $n_{\|}$is the parallel refractive index. To include first order corrections in equilibrium gradients, we use the susceptibility given by [3]

$$
\chi_{x x}\left(x, k_{x}\right)=\left(1-\frac{i}{2} \frac{\partial}{\partial k_{x}} \frac{\partial}{\partial x}\right) \chi_{x x}^{\text {HOMO }}\left(x, k_{x}\right)
$$

where the second term in the bracket is the first order correction term and

$$
\chi_{x x}^{\text {HOMO }}\left(x, k_{x}\right)=\sum_{j=e, D} \frac{\omega_{p, j}^{2}}{\omega}\left(\frac{1}{\omega+\Omega_{j}}+\frac{1}{\omega-\Omega_{j}}\right) e^{-\lambda_{j}} \frac{I_{1}\left(\lambda_{j}\right)}{\lambda_{j}},
$$

is the hot susceptibility tensor for homogeneous media given by Stix $[1,2], \omega_{p, j}$ is the plasma frequency, $\Omega_{j}$ is cyclotron frequency, $I_{1}$ is the modified Bessel function of order 1 and $\lambda_{j}=0.5 k_{x}^{2} \rho_{L, j}^{2}$ where $\rho_{L, j}$ is the Larmor radius. We have assumed that $\omega \ll \Omega_{D}, \Omega_{e}$ so that $K_{x y} \approx 0$. The tensor is Hermitian (no absorption) and the wave does not propagate in the $y$ direction. The kinetic energy flux is given by $[1,2]$

$$
T_{x}(x)=-\frac{\omega \epsilon_{0}}{4} E_{x}^{*}\left(\frac{d}{d k_{x}} K_{x x}\right) E_{x} .
$$

\subsection{Fast wave minority heating}

The up- and downshift in the parallel wave number is here studied for a fast wave minority heating scenario in a plasma of electrons, deuterium and hydrogen minority ions. The geometry can be described using a Cartesian coordinate system, $(x, y, z)$, where the plasma is homogeneous in the $y$ and $z$ directions. The magnetic field is described by

$$
\mathbf{B}(x)=B(x)\left(\cos \alpha \boldsymbol{e}_{z}+\sin \alpha \boldsymbol{e}_{x}\right),
$$

where $\alpha$ is the angle between the $z$-axis and parallel direction. Neglecting the parallel electric field, the wave equation for the fast wave in a Maxwellian plasma is given by $[1,2]$

$$
\mathbf{M E}_{\perp}-\frac{\omega^{2}}{c^{2}} L\left[\mathbf{K}_{0}\left(x, k_{X}\right), \mathbf{E}_{\perp}(x)\right]=\mathbf{0},
$$

where

$$
\begin{gathered}
\mathbf{M}=\left[\begin{array}{cc}
k_{z}^{2} \cos ^{2} \alpha & 0 \\
0 & k_{z}^{2}-\frac{\partial^{2}}{\partial x^{2}}
\end{array}\right], \\
\mathbf{K}_{\mathbf{0}}=\mathbf{K}_{\text {cold }}+\chi_{H},
\end{gathered}
$$

$$
\begin{gathered}
\mathbf{K}_{\text {cold }}=\mathbf{I}-\sum_{j=e, D} \frac{\omega_{p, j}}{\omega\left(\omega^{2}-\Omega_{j}^{2}\right)}\left[\begin{array}{cc}
\omega & i \Omega_{j} \\
-i \Omega_{j} & \omega
\end{array}\right], \\
\chi_{\mathrm{H}}=\frac{1}{2} \frac{\omega_{p, H}^{2}}{\omega}\left(\frac{Z(\zeta)}{k_{\|} v_{t h}}\left[\begin{array}{cc}
1 & i \\
-i & 1
\end{array}\right]-\frac{1}{\omega+\Omega_{H}}\left[\begin{array}{cc}
1 & -i \\
i & 1
\end{array}\right]\right), \\
\zeta=\frac{\omega-\Omega_{H}+k_{\|} v_{p}}{k_{\|} v_{t h}} \\
k_{\|}=k_{Z} \cos \alpha+k_{x} \sin \alpha,
\end{gathered}
$$

where $Z(\zeta)$ is the plasma dispersion function [1,2], $v_{t h}$ is the thermal velocity and $v_{p}$ is the mean plasma fluid velocity. The local absorption is given by $[1,2]$

$$
P(x)=\omega \epsilon_{0} \mathbf{E}_{\perp}{ }^{*} \mathbf{K}_{0}^{A} \mathbf{E}_{\perp}
$$

where $\mathbf{K}_{0}^{A}$ is the anti-Hermitian part of the dielectric tensor.

\section{Wavelet representation of the wave equation}

The dielectric response tensors in Eqs. (2), (4) and (7) are Fourier transformed in space. To represent the dielectric response using a complex Morlet wavelet representation, we use the following equation $[3,4]$

$$
\begin{gathered}
L[\mathbf{K}(x, k), \mathbf{E}(x)]=\mathbf{W T}^{-1}\left[\mathbf{K}_{a, b}(x) \mathbf{E}_{a, b}\right](x) \quad(10) \\
\mathbf{K}_{a, b}(x)=\mathbf{K}\left(x, \frac{\sigma}{a}\right)+\left.i X \frac{\partial \mathbf{K}}{\partial k}\right|_{\frac{\sigma}{a}}+\left.\frac{1}{2 a^{2}}\left(1-X^{2}\right) \frac{\partial^{2} \mathbf{K}}{\partial k^{2}}\right|_{\frac{\sigma}{a}}
\end{gathered}
$$

where $\mathbf{W} \mathbf{T}^{-1}$ is the inverse wavelet transform, $a$ and $b$ are the wavelet scale and dilatation parameters, $\sigma=6$ is a constant, $X=(x-b) / a$ and

$$
\mathbf{E}_{a, b}=\mathbf{W T}[\mathbf{E}(x)](a, b),
$$

is the wavelet transform of the electric field [11].

\subsection{Wavelet representation of the kinetic Alfvén wave equation}

To construct an iterative solution for Eq. (4) we move the dispersive terms to the right hand side and use Eq. (10) to obtain

$$
\left(n_{\|}^{2}-1-\chi_{e}\right) E_{x}=\mathbf{W T}^{-1}\left[\chi_{a, b}^{i}(x) E_{a, b}\right](x) .
$$

Since the left hand side is algebraic, a fix-point formulation can be constructed

$$
E_{x}^{(n+1)}=\frac{1}{n_{\|}^{2}-1-\chi_{e}} \mathbf{W} \mathbf{T}^{-1}\left[\chi_{a, b}^{i}(x) E_{a, b}^{(n)}\right](x) .
$$

This equation tend to be unstable. However, stable solutions can be found using the Anderson Acceleration scheme [10]. 


\subsection{Wavelet representation of the fast wave minority heating equation}

In this study, the curl-curl operator in Eq. (7) is solved using the iterative wavelet spectral method. By Fourier transforming the curl-curl operator in the $x$ direction, we can rewrite Eq. (7) as

$$
L\left[\frac{c^{2}}{\omega^{2}} \mathbf{M}_{k}\left(k_{x}\right)+\mathbf{K}_{0}\left(x, k_{x}\right), \mathbf{E}_{\perp}(x)\right]=\mathbf{0} .
$$

By using Eq. (10) and Anderson Acceleration scheme, the solution is given by

$$
\mathbf{E}_{\perp}^{(n+1)}=-\mathbf{K}_{c o l d}^{-1} \cdot \mathbf{W} \mathbf{T}^{-1}\left[\left(\frac{c^{2}}{\omega^{2}} \mathbf{M}_{a, b}^{k}+\chi_{a, b}^{H}\right) \mathbf{E}_{\perp, a, b}^{(n)}\right](x) .
$$

\section{Results}

\subsection{Kinetic Alfvén wave}

The solution to the kinetic Alfvén wave was obtained on an interval $x=[2,8]$ using the following parameters; $B=1.32+0.1 \cos (-\pi(x-2) / 6) \quad \mathrm{T}, \quad T_{e}=1 \mathrm{keV}$, $n_{e}=5 e 19 \mathrm{~m}^{-3}, k_{z}=1 \mathrm{~m}^{-1}$ and a wave frequency of 500 $\mathrm{kHz}$. The wave is excited on the left boundary and propagates to the right. The wave is absorped at the right boundary (no reflection) and reflections due to inhomogeneities are neglected.

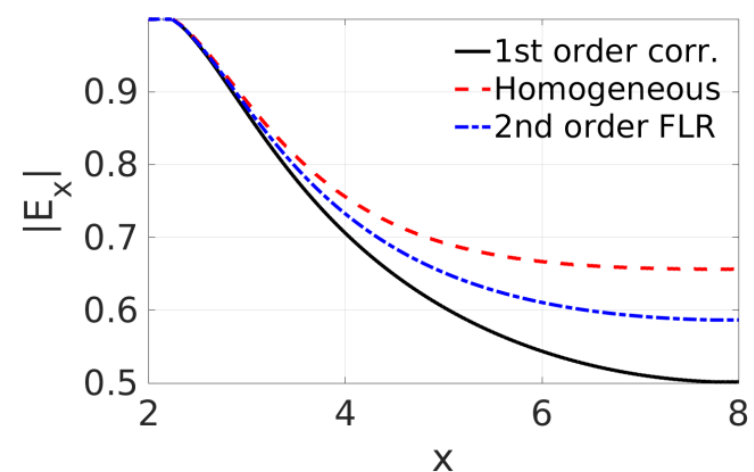

Fig. 1. Amplitude of the electric field $E_{x}$. The solid black curve is the solution to Eq. (4). The red dashed and blue dasheddotted solutions are obtained using $K_{x x}=1+\chi_{x x}^{H O M O}$. FLR effects have been expanded to order 2 for the blue dasheddotted solution.

The amplitude of the solution to Eq. (4) is shown in Figure 1 (black solid line). The solution is compared to the red dashed and blue dashed-dotted solutions, which are obtained assuming a homogeneous dielectric response tensor, i.e $K_{x x}=1+\chi_{x x}^{\text {HOMO }}$. The FLR effects are expanded to order 2 in the blue dashed-dotted solution.

As the wave propagates, the rate of amplitude decay depends on the dielectric tensor model used in the wave equation. The solution to Eq. (4) has the fastest decay in amplitude, while the solutions using a homogeneous response tensor have a weaker amplitude decay.

Figure 2 shows the kinetic energy flux of the three wave solutions. The solution to Eq. (4) show a constant energy flux, i.e. conserves the energy (energy flux difference between left and right boundary is $\sim 1 \%$ ). The other two solutions show a strongly increasing trend, i.e. energy is not conserved.

Figure 3 shows the wavelet spectrum of the wave solution. The wavelet scale parameter and wave number are related through $a=\sigma / k_{x}$. The figure shows how the spectrum is localized near values that correspond to the dispersion relation of the kinetic Alfvén wave.

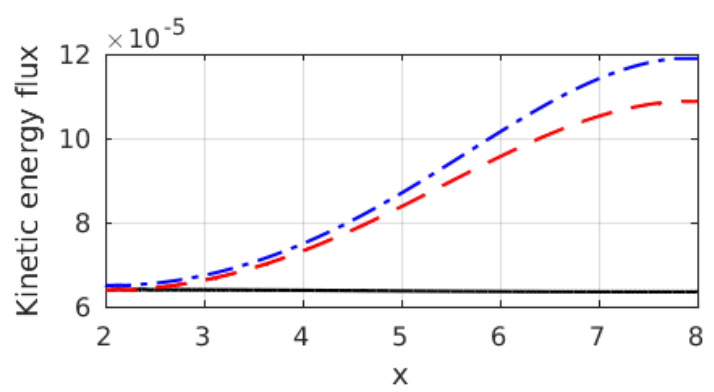

Fig. 2. Kinetic energy flux as a function of $x$. Same color coding as Figure 1. Conservation of the energy is only acquired using first order corrections in the dielectric response tensor.

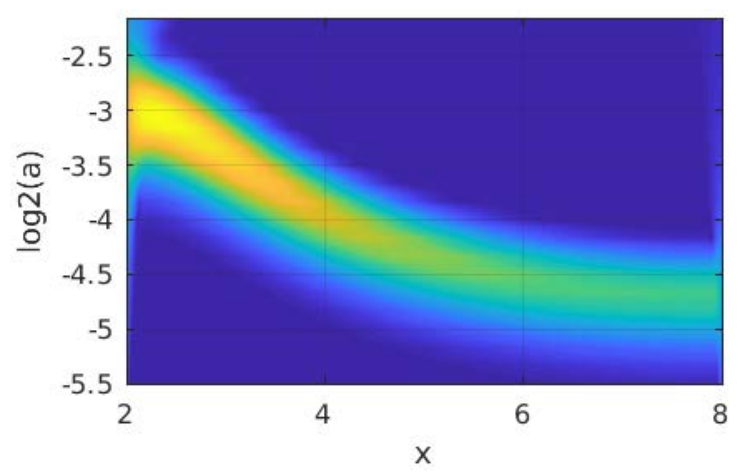

Fig 3. Wavelet spectra of $E_{x}$ component of the kinetic Alfven wave. The scale vector is related to the wave number through $a=\sigma / k_{X}$.

\subsection{Fast wave minority heating}

The solution to the fast wave was obtained on an interval $x=[2,4]$ using the following parameters; magnetic field $B=B_{0} R_{0} / x, B_{0}=3.8 \mathrm{~T}, R_{0}=3 \mathrm{~m}, T_{e}=8 \mathrm{keV}$, $n_{e}=4 e 19 \mathrm{~m}^{-3}, n_{\phi}=25, \alpha=0.051 \mathrm{rad}$ with a wave frequency of $51.1 \mathrm{MHz}$ and hydrogen minority concentration of $1.5 \%$. To separate the location of the absorption of the incident and reflected waves, a finite equilibrium velocity is included, $v_{p}=8 e 6 \mathrm{~m} / \mathrm{s}$. The cold hydrogen cyclotron resonance (i.e. $\omega=\Omega_{H}$ ) is located at $r=3.43 \mathrm{~m}$, while the Doppler shifted cyclotron resonance (i.e. $\omega=\Omega_{H}+k_{\|} v_{p}$ ) is located near $r=2.82$ $\mathrm{m}$ when excluding the up- and downshift in the parallel wave number.

The solution to Eq. (7) is shown in Figure 4. The fast wave is excited using a boundary condition on the right side and propagates to the left (see blue curve). The wave is reflected at the left boundary and propagates back to the right boundary (see red curve), were it is absorbed. The fast wave will therefore pass the ion cyclotron resonance twice, but with different $k_{\|}$. 
The deuterium resonance is located near $r=1.7 \mathrm{~m}$, i.e. outside the simulated interval. As the wave approaches the deuterium resonance, the solution in Figure 4 verifies that the amplitude for $E_{+}$decays due to screening by the deutrons.

Similarly, the hydrogen ions tend to screen $E_{+}$near the Doppler shifted resonance, causing a local minimum. Figure 4 shows that the minima for the incident and reflected waves are located at 3.0 and $2.8 \mathrm{~m}$ respectively. The difference in location is due to the up- and downshift in $k_{\|}$of the two waves.
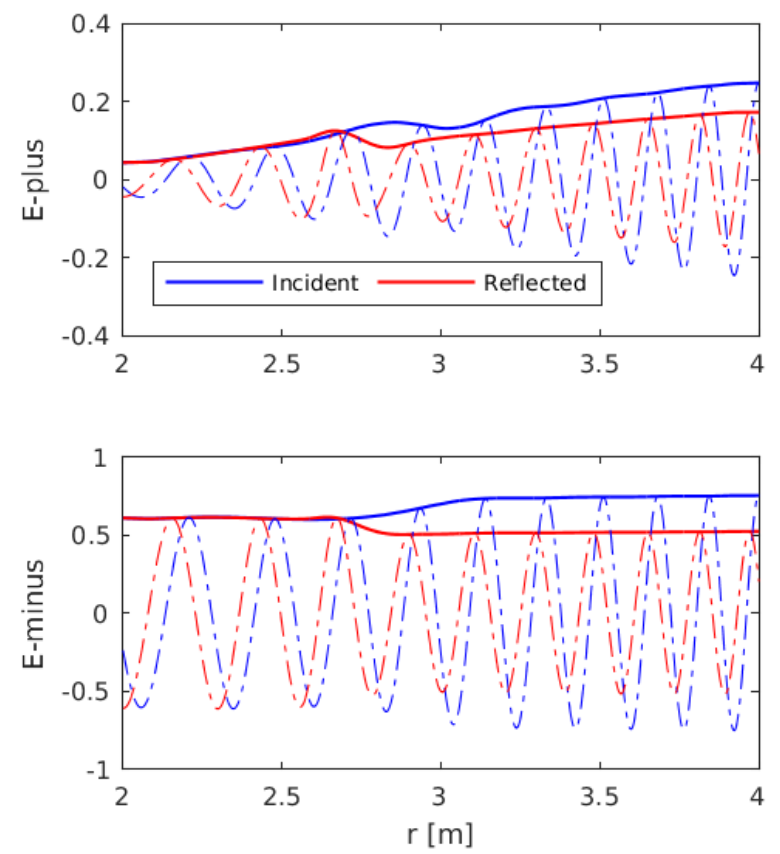

Fig 4. Left and right hand circular wave components of the fast wave. Solid and dashed-dotted curves show the amplitude and real part of the wave solutions. The wave is excited at the right boundary (blue) and propagates to the left boundary where it is reflected (red). The reflected wave propagates back to the right boundary, were it is absorbed.

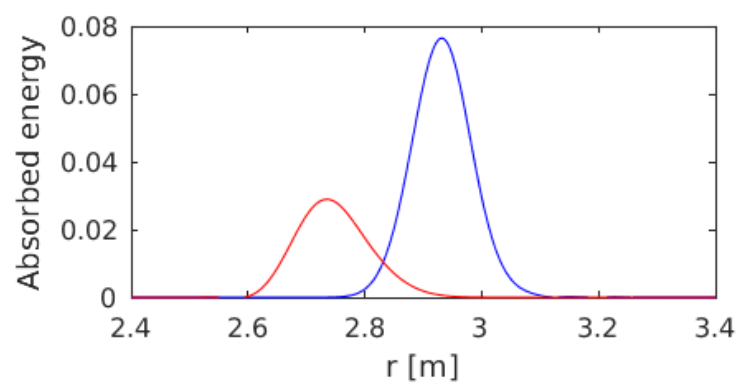

Fig 5. Absorbed energy of the incident (blue) and reflected (red) wave.

The absorption of energy is shown in Figure 5. The absorption peak of the incident wave is located at 2.93 $\mathrm{m}$, while the reflected wave have the peak at $2.74 \mathrm{~m}$. This shift in the absorption is a direct consequence of the up- and downshift of $k_{\|}$. Another effect of is the different full width at half maximum (FWHM) values. The FWHM values are $0.12 \mathrm{~m}$ and $0.14 \mathrm{~m}$ for the incident and reflected waves respectively.

\section{Discussion and conclusions}

In this study we have shown that this new iterative scheme is capable of solving spatially dispersive wave equation for inhomogeneous media.

The method has been used to study the energy flux of a propagating kinetic Alfvén wave to all orders in FLR. The results show that in order to conserve the kinetic energy flux, first order corrections in equilibrium gradients must be included. The solution using the homogeneous dielectric tensor (no first order corrections) did not conserve the energy. Note that the solution to the wave equation using a second order expansion in FLR effects gives a better amplitude solution compared to the all-order solution without first order corrections. To conclude, first order corrections in the equilibrium gradients are essential for accurate solutions and energy conservation in inhomogeneous plasma.

In the second case, the method has been successfully applied to a fast wave minority scenario to study the upand downshift of the parallel wave number. The method is capable of separating the responses for the incident and reflected waves and account for the up- and downshift of $k_{\|}$, which is difficult to do with FEM.

The calculation of the fast wave heating model took about 200 iterations. The reason is that the curl-curl operator is included iteratively. The performance is expected to improve by inverting the curl-curl operator with a finite element scheme and use the iterative wavelet spectral scheme to include the spatial dispersion.

\section{References}

1. T.H. Stix, Waves in plasmas (AIP, New York, 1992)

2. D.G. Swanson, Plasma Waves $2^{\text {nd }}$ ed., (IOP Publishing, 2009)

3. T. Hellsten, T. Johnson, P. Vallejos, Journal of Physics: Conference Series 561012010 (2014)

4. P. Vallejos, T. Johnson, T. Hellsten, Journal of Physics: Conference Series 775012016 (2016)

5. R.J. Dumont, C.K. Phillips, D.N. Smithe, Phys. of Plasmas 12042508 (2005)

6. D.N. Smithe, Plasma Phys. Control. Fusion 311105 (1989)

7. J.Hedin et al, Nuclear Fusion 42 527-540 (2002)

8. L. Villard et al, Comp. Phys. Reports 495 (1986)

9. D.L. Green, L.A. Berry, Comput. Phys. Commun. 185736 (2014)

10. H.F. Walker, P. Ni, SIAM J Numer. Anal. 491715 (2011)

11. I. Daubechies Ten lectures on wavelets (Philadelphia, PA, USA: Society for Industrial and Applied Mathematics) (1992) 